\title{
Sensibilité au triadiménol et au fenpropimorphe de populations françaises d'Erysiphe graminis DC f. sp. hordei Marchal
}

\author{
Didier ANDRIVON $\left({ }^{1}\right)$, Eckhard LIMPERT $\left({ }^{*}\right)$ \& Friedrich Georg FELSENSTEIN $\left({ }^{*}\right)$ \\ I.N.R.A., Laboratoire d'Epidémiologie, Chaire de Pathologie végétale, INA-PG Centre de Recherches de \\ Paris-Grignon, F 78850 Thiverval Grignon \\ (*) Technische Universität München, Lehrstuhl für Pflanzenbau und Pflanzenzüchtung, D-8050 Freising- \\ Weihenstephan, RFA
}

Une proportion importante d'isolats d'Erysiphe graminis f. sp. hordei résistants au triadiménol a été mise en évidence dans les populations du Nord de la France et de Champagne en 1986, confirmant des observations réalisées en 1985. Les populations du Languedoc et de l'Ouest du pays présentent, pour leur part, des niveaux de sensibilité nettement supérieurs à ceux observés dans les autres régions. Une légère modification de la sensibilité de la population champenoise au fenpropimorphe a également été observée ; aucune résistance croisée entre triadiménol et fenpropimorphe n'est apparue.

Mots clés additionnels : Fongicides, inhibiteurs des stérols, résistance croisée, oidium, orge. hordei Marchal.

Significant proportions of barley mildew isolates resistant to triadimenol have been found in populations from northern France and Champagne in 1986, confirming observations made in 1985. Populations from Languedoc and western France showed sensitivity levels considerably higher than those observed in the other two regions. A slight modification of the sensitivity to fenpropimorph has also been found in the population from Champagne ; no cross resistance was noted between triadimenol and fenpropimorph.

Additional key words : Fungicides, sterol biosynthesis inhibitors, cross resistance, barley, mildew.

\section{INTRODUCTION}

L'oïdium de l'orge, dû à Erysiphe graminis DC f. $\mathrm{sp}$. hordei Marchal, est susceptible de causer des pertes de rendement non négligeables, estimées par LIMPERT (1985) à une moyenne de 7 p. 100 par an pour l'ensemble des pays de la C.E.E. En France, la lutte contre ce parasite repose essentiellement sur une protection fongicide des cultures, en traitement des semences (triadiménol) comme en végétation (triadiménol, triadiméfon, propiconazole, dichlobutrazol, flutriafol, fenpropimorphe...).

Bien que divers auteurs (FUCHS \& DRANDAREVSKI, 1976 ; GASZTONYI \& JOSEPOVITS, 1984) considèrent comme improbable le développement en conditions

(') Adresse actuelle : Technische Universität München, Lehrstuhl für Pflanzenbau und Pflanzenzüchtung, D-8050 Freising-Weihenstephan, RFA. naturelles de populations résistantes aux inhibiteurs de la biosynthèse des stérols, groupe auquel appartiennent les matières actives mentionnées plus haut, plusieurs exemples montrent qu'un tel développement est néanmoins possible, tant en serre qu'au champ (FLETCHER \& WOLFE, 1981 ; BUTTERS et al., 1984 ; LIMPERT, 1985 ; SCHEPERS, 1985 ; SHERIDAN et al., 1985). Dans le cas de l'oïdium de l'orge, des phénomènes de résistance à plusieurs inhibiteurs de la $\mathrm{C}-14$ déméthylation des stérols ont été rapportés en Grande-Bretagne (FLETCHER \& WOLFE, 1981 ; BUTTERS et al., 1984) et en R.F.A. (LIMPERT \& SCHWARZBACH, 1981 ; LIMPERT, 1985).

L'intensité de la sélection exercée par suite de l'emploi massif et répété de ce type de fongicides, de même que la rapidité du déplacement des populations d'oïdium dans la direction des vents dominants (LIMPERT, 1985), pouvaient justifier la crainte d'apparition de cas de résistance également en France. De fait, 
dès 1985 , une baisse de sensibilité au triadiménol dans les populations de Lorraine et du Nord de la France ont été mises en évidence par des analyses de laboratoire (LIMPERT, 1986). Le présent article décrit les résultats de l'étude du comportement de populations d'Erysiphe graminis f. sp. hordei, piégées en 1986 dans 4 régions françaises, vis-à-vis de 2 inhibiteurs de la synthèse des stérols à site d'action différents : le triadiménol, inhibiteur de la C-14 déméthylation, et le fenpropimorphe, inhibiteur de la $\Delta 8-\Delta 7$ isomérisation et/ou de la $\Delta 14$ réduction.

\section{MATÉRIEL ET MÉTHODES}

Les analyses ont été effectuées sur des isolats monopustules d'Erysiphe graminis f. sp. hordei, issus de spores collectées de manière continue directement dans l'air lors de la traversée des régions étudiées. Ces piégeages ont été réalisés sur la lignée universellement sensible S.M. 4142, au moyen d'un piège à spores Schwarzbach* (SCHWARZBACH, 1979) fixé sur le toit d'une voiture. Les méthodes de piégeage mobile et d'analyse sont décrites de manière plus détaillée par LIMPERT et al. (1984) et par LIMPERT (1985, 1986).

Quatre régions, choisies pour leurs caractéristiques quant à la culture de l'orge, ont été étudiées : le Languedoc (Beaucaire - Montpellier) et l'Ouest (Le Blanc Poitiers - Ancenis - Le Mans) représentent des conditions d'intensification faible à modérée (usage des fongicides limité à moins d'un traitement par ha et par an), alors que le Nord (Mons - Lille - Calais) et surtout la Champagne (Reims - Ste Menehould) comptent parmi les régions les plus intensives du pays (2 à 3 traitements fongicides par ha et par an) (ANONYME, 1986).

Des lots de graines de la variété d'orge «Igri » (portant le gène de résistance $M l-r a$, surmonté par tous les isolats testés) ont été traités avec une gamme de doses en progression géométrique de raison 2 , et comprises entre 0,73 et $750 \mathrm{mg} \cdot \mathrm{kg}^{-1}$ pour le triadimé-

* Fabriqué par BuRkard Manufacturing Co. Ltd., Rickmansworth, Herts. WD3 1PJ, England. nol (Baytan S, 25 p. 100 de triadiménol, 3 p. 100 de fubéridazole ; cette formulation donne des résultats ne différant pas de ceux obtenus avec le triadiménol seul (LIMPERT, non publié)), et entre 0,59 et $75 \mathrm{mg} \cdot \mathrm{kg}^{-1}$ pour le fenpropimorphe (BAS $42103 \mathrm{~F}, 40$ p. $100 \mathrm{de}$ fenpropimorphe). Les fongicides utilisés ont été aimablement fournis par leurs producteurs respectifs. Dans le cas du fenpropimorphe, non homologué pour traitement de semences, la formulation utilisée permet de les réaliser pour les besoins du test.

Après culture en pots des graines traitées ( 10 jours, $19^{\circ} \mathrm{C}, 92 \mu \mathrm{E} \cdot \mathrm{m}^{-2} \cdot \mathrm{s}^{-1}$, éclairage permanent), trois segments $(12 \times 5 \mathrm{~mm})$ ont été prélevés dans la partie médiane de la première feuille des plantules ainsi obtenues, et mis en survie, dans des boîtes compartimentées, sur milieu agar $(0,5 \mathrm{p}$. 100) supplémenté avec du benzimidazole (15 $\left.\mathrm{mg} . \mathrm{l}^{-1}\right)$. Chaque compartiment reçoit une gamme complète (soit un segment par dose, plus un segment témoin non traité) et est inoculé, à l'aide d'une tour miniature, avec les spores issues d'une seule colonie d'oïdium (densité d'inoculation 300-400 spores $\mathrm{cm}^{-2}$ ). Pour chaque série d'inoculations, un témoin, constitué de 4 isolats récoltés au champ avant la commercialisation de chacun des 2 produits, a également été inoculé. Dans le cas du fenpropimorphe, la réaction étant moins stable que vis-à-vis du triadiménol (LIMPERT, non publié), une répétition a été effectuée pour chaque isolat testé. Après incubation ( 7 jours, $17{ }^{\circ} \mathrm{C}, 5,5 \mu \mathrm{E} . \mathrm{m}^{-2} \mathrm{~s}^{-1}$, éclairage permanent), la dose la plus élevée permettant un degré d'attaque d'au moins 50 p. 100 par rapport au segment témoin a été notée pour chaque isolat. Le facteur de résistance a été calculé en rapportant cette dose à celle correspondante pour les isolats sensibles standard $\left(1,46 \mathrm{mg} \cdot \mathrm{kg}^{-1}\right.$ pour le triadiménol ; 18,75 $\mathrm{mg} . \mathrm{kg}^{-1}$ pour le fenpropimorphe).

\section{RÉSULTATS}

La répartition des isolats testés en classes de sensibilité au triadiménol dans les 4 régions étudiées est résumée dans le tableau 1. Alors que la sensibilité des populations du Languedoc est peu différente de celle

TABLEAU 1

Répartition en classes de résistance au triadiménol d'isolats d'oidium de l'orge piégés dans quatre régions françaises. Distribution into triadimenol-resistance classes of barley mildew isolates trapped in four French regions in 1986.

\begin{tabular}{|c|c|c|c|c|c|c|c|c|c|c|c|}
\hline \multirow[t]{2}{*}{$\begin{array}{l}\text { Région d'origine des isolats } \\
\quad \text { (itinéraire de piégeage) }\end{array}$} & \multirow{2}{*}{$\begin{array}{l}\text { Nombre d'isolats } \\
\text { analysés } \\
\text { I } \\
\text { II }\end{array}$} & \multicolumn{10}{|c|}{$\begin{array}{c}\text { Pourcentage d'isolats par dose de triadiménol (mg m.a./kg semences) (I) } \\
\text { et facteur de résistance correspondant (II) }\end{array}$} \\
\hline & & 1,46 & $\begin{array}{l}2,9 \\
2\end{array}$ & $\begin{array}{l}5,9 \\
4\end{array}$ & $\begin{array}{c}11,7 \\
8\end{array}$ & $\begin{array}{l}23,4 \\
16\end{array}$ & $\begin{array}{l}46,7 \\
32\end{array}$ & $\begin{array}{l}93,7 \\
64\end{array}$ & $\begin{array}{l}187,5 \\
128\end{array}$ & $\begin{array}{l}375 \\
256\end{array}$ & $\begin{array}{l}750 \\
512\end{array}$ \\
\hline Témoin & 4 & 100 & 0 & 0 & 0 & 0 & 0 & 0 & 0 & 0 & 0 \\
\hline $\begin{array}{l}\text { Languedoc } \\
\text { (Beaucaire - Montpellier) }\end{array}$ & 24 & 33,3 & 50,0 & 12,5 & 0 & 0 & 4,2 & 0 & 0 & 0 & 0 \\
\hline $\begin{array}{l}\text { Ouest } \\
\text { (Le Blanc - Poitiers - Ancenis } \\
\text { Le Mans) } \\
\text { Champagne }\end{array}$ & 33 & 0 & 12,1 & 9,1 & 12,1 & 45,5 & 18,2 & 3,0 & 0 & 0 & 0 \\
\hline (Reims - Ste-Menehould) & 35 & 0 & 0 & 0 & 2,9 & 2,9 & 8,6 & 82,7 & 2,9 & 0 & 0 \\
\hline $\begin{array}{l}\text { Nord } \\
\text { (Mons (frontière) - Lille - Calais) }\end{array}$ & 46 & 0 & 0 & 8,7 & 28,3 & 10,9 & 13,0 & 13,0 & 17,4 & 6,5 & 2,2 \\
\hline
\end{tabular}


TABLEAU 2

Répartition en classes de résistance au fenpropimorphe d'isolats d'oïdium de l'orge piégés en 1986 dans trois régions françaises (moyenne de deux répétitions).

Distribution into fenpropimorph-resistance classes of barley mildew isolates trapped in three French regions in 1986 (mean of two replicates).

\begin{tabular}{|c|c|c|c|c|c|c|}
\hline \multirow{2}{*}{$\begin{array}{l}\text { Région d'origine des isolats } \\
\text { (itinéraire de piégeage) }\end{array}$} & \multirow{2}{*}{$\begin{array}{c}\text { Nombre d'isolats } \\
\text { analysés } \\
\text { I } \\
\text { II }\end{array}$} & \multicolumn{5}{|c|}{$\begin{array}{l}\text { Pourcentage d'isolats par dose de fenpropimorphe (mg m.a. } / \mathrm{kg} \text { semences) (I) } \\
\text { et facteur de résistance correspondant (II) }\end{array}$} \\
\hline & & $\begin{array}{l}4,69 \\
0,25\end{array}$ & $\begin{array}{l}9,37 \\
0,5\end{array}$ & $\begin{array}{l}18,75 \\
1\end{array}$ & $\begin{array}{c}37,5 \\
2\end{array}$ & $\begin{array}{c}75,0 \\
4\end{array}$ \\
\hline Témoin & 4 & 0 & 0 & 100 & 0 & 0 \\
\hline $\begin{array}{l}\text { Languedoc } \\
\text { (Beaucaire - Montpellier) }\end{array}$ & 13 & 15,4 & 23,1 & 50,0 & 11,5 & 0 \\
\hline $\begin{array}{l}\text { Nord } \\
\text { (Mons (frontière) - Lille - Calais) }\end{array}$ & 36 & 1,4 & 23,6 & 58,3 & 9,7 & 6,9 \\
\hline $\begin{array}{l}\text { Champagne } \\
\text { (Reims - Ste-Menehould) }\end{array}$ & 36 & 0 & 1,4 & 51,4 & 40,3 & 6,9 \\
\hline
\end{tabular}

des isolats standard, quoique légèrement supérieure, une modification sensible du niveau moyen de résistance se manifeste dans chacune des 3 autres régions. En Champagne, une très grande majorité d'isolats présente un facteur de résistance identique et élevé (64). De tels isolats restent rares dans l'Ouest mais sont relativement fréquents dans le Nord. Dans cette dernière région, la distribution de la population n'est pas unimodale : le premier maximum est peu différent de celui rencontré dans l'Ouest, alors que le second est similaire à celui observé dans le Sud-Est de l'Angleterre (LIMPERT et al., non publié). Ceci laisse supposer un apport des fractions les plus résistantes de la population du Nord de la France par les vents de Nord-Ouest, et des fractions les plus sensibles par les vents de Sud-Ouest.

La sensibilité des populations d'oïdium au fenpropimorphe (tabl. 2) est en moyenne peu différente d'une région à l'autre, et est également très proche de celle des isolats standard. Toutefois, un test de $\chi^{2}$ montre une déviation légère mais significative des populations champenoises vers des sensibilités réduites, sans que les performances du produit au champ n'aient paru altérées (ARCHIMBAUD, SIMON, comm. pers.). D'autre part, la comparaison de la sensibilité des isolats, tant de Champagne que du Nord, au triadiménol et au fenpropimorphe n'a pas montré l'existence d'une résistance croisée entre ces 2 matières actives.

\section{DISCUSSION}

Niveaux d'intensification (mesurés par la fréquence d'emploi des fongicides) et de sensibilité des populations d'oïdium au triadiménol apparaissent clairement liés sur l'ensemble des 4 régions françaises étudiées. Les résultats des analyses au laboratoire montrent l'existence dans plusieurs de ces régions de parties importantes des populations du parasite résistant à ce fongicide. En Champagne, région d'où nous sont parvenus des échantillons d'oïdium récoltés au champ et ayant survécu à diverses applications à base d'inhibiteurs de la C-14 déméthylation des stérols, l'abondance d'isolats présentant un même facteur de résistance (64) laisse penser que cette fraction de la popu- lation est à l'origine de la baisse d'efficacité de ce groupe de produits dans le contrôle de la maladie. Le facteur de résistance de 64 , mesuré dans les conditions de notre test, pourrait donc être considéré comme marquant la limite d'efficacité du triadiménol au champ à la dose recommandée en France. Bien qu'une réduction d'efficacité soit également vraisemblable sur les isolats à facteur de résistance sensiblement inférieur à ce seuil (LIMPERT, 1985), une estimation du risque minimal encouru par les cultures d'orge traitées dans une région donnée peut être réalisée en considérant l'importance dans cette région de la fraction de la population d'oïdium dont le facteur de résistance égale ou dépasse 64 .

Compte tenu de la résistance croisée positive existant chez Erysiphe graminis f. sp. hordei entre tous les inhibiteurs de la C-14 déméthylation des stérols (HOLLOMON, 1982 ; BUTTERS et al., 1984), l'existence d'une résistance au triadiménol chez de larges proportions des populations d'oïdium du Nord et de l'Est de la France signifie également que plusieurs autres matières actives (triadiméfon, propiconazole, dichlobutrazol, flutriafol...) sont elles aussi concernées par ce phénomène, ce qui restreint considérablement les possibilités de lutte chimique dans ces régions. Par contre, l'absence d'une telle résistance croisée entre inhibiteurs de la C-14 déméthylation et inhibiteurs de la $\Delta 8-\Delta 7$ isomérisation et/ou de la $\Delta 14$ réduction (fenpropimorphe, tridémorphe, fenpropidine), confirmée par nos observations, permet l'emploi de ce dernier groupe de fongicides dans les zones touchées par la résistance au triadiménol. Il n'est toutefois pas possible d'exclure le risque d'émergeance, à plus ou moins long terme, d'isolats résistant à ces composés. Aussi, afin de préserver leur efficacité actuelle, un raisonnement des traitements et une intégration plus poussée des diverses méthodes de lutte disponibles semblent des précautions indispensables.

Reçu le 14 octobre 1986. Accepté le 12 mars 1987.

\section{REMERCIEMENTS}

Les auteurs remercient la Commission des Communautés Européennes pour son concours financier à cette étude. 


\section{RÉFÉRENCES BIBLIOGRAPHIQUES}

Anonyme, 1986. Enquête céréalière par sondage 1985 : techniques. ONIC, Paris, 39 p.

Butters J., Clark J., Hollomon D. W., 1984. Resistance to inhibitors of sterol biosynthesis in barley powdery mildew. Med. Fac. Landbouww. Rijkuniv. Gent, 49 (2a), 143-151.

Fletcher J. T., Wolfe M. S., 1981. Insensitivity of Erysiphe graminis f. sp. hordei to triadimefon, triadimenol and other fungicides. Proc. 1981 Brit. Crop Prot. Conf., Pests \& Diseases, 633-640.

Fuchs A., Drandarevski C. A., 1976. The likelihood of development of resistance to systemic fungicides which inhibit ergosterol biosynthesis. Neth. J. Plant Pathol., 82, 85-87.

Gasztonyi M., Josepovits G., 1984. Metabolism of some sterol inhibitors in fungi and higher plants, with special reference to the selectivity of fungicidal action. Pestic. Sci., 15, 48-55.

Hollomon D. W., 1982. The effects of tridemorph on barley powdery mildew : its mode of action and cross sensitivity relationships. Phytopathol. Z., 105, 279-287.

Limpert E., 1985. Ursachen unterschiedlicher Zusammensetzung des Gerstenmehltaus, Erysiphe graminis $D C f$. sp. hordei Marchal ; und deren Bedeutung für Züchtung und Anbau von Gerste in Europa. These Doct. Sci. Nat., Univ. Tech. Munich, 189 p.
Limpert E., 1986. L'oïdium de l'orge : quelles races en France? Persp. Agric., 105, 35-39.

Limpert E., Schwarzbach E., 1981. Virulence analysis of barley powdery mildew in different European regions in 1979 and 1980. Barley Genetics IV, Proc. 4th Int. barley Genet. Symp., Edinburgh, 1981 : 458-465.

Limpert E., Schwarzbach E., Fischbeck G., 1984. Influence of weather and climate on epidemics of barley mildew, p. 146-157. In H. Lieth, R. Fantechi \& Schnitzler, eds., Interactions between climate and biosphere, Progress in biometeorology, vol. 3, Swets \& Zeitlinger B.V., Lisse, 392 p.

Schepers H. T. A. M., 1985. Development and persistance of resistance to fungicides in Sphaerotheca fuliginea in cucumbers in the Netherlands. 1 hese Dout. Sci. Agron., Univ. W ageningen, $56 \mathrm{p}$.

Schwarzbach E., 1979. A high throughput jet spore trap for collecting mildew spores on living leaves. Phytopathol. Z., 94 : 165-171.

Sheridan J. E., Grbavac N., Sheridan M. H., 1985. Triadimenol insensitivity in Pyrenophora teres. Trans. Brit. mycol. Soc., 85 : 338-341. 